\title{
Multiple-unit activity of the rabbit cerebral cortex in single-session avoidance conditioning*
}

\author{
MICHAEL GABRIEL $\dagger$, WILLIAM WHEELER, and RICHARD F. THOMPSON \\ Department of Psychobiology, University of California, Irvine, California 92664
}

\begin{abstract}
Multiple-unit neuronal activity was recorded from three "nonspecific" polysensory regions of the cerebral cortex of rabbit during single-session discrete-trial avoidance acquisition and during asymptotic conditioned response (CR) performance (overtraining). The CS was a pure tone, and the UCS was a shock delivered through the grid floor of the rotating-wheel conditioning apparatus. The CS did not evoke unit activity in any of the areas on the initial conditioning trials. Two of the cortical areas (motor-sensory cortex and dorsal limbic cortex) showed significant increases in CS-evoked multiple-unit activity over stages of acquisition, paralleling the development of behavioral CRs. The same two areas also yielded substantial CS-evoked unit activity during overtraining. In overtraining, an analysis of four consecutive 50-msec post-CS-onset periods revealed a decline in multiple-unit activity over periods within motor-sensory cortex, whereas dorsal limbic cortex showed a more sustained unit response over the four periods. A third nonspecific cortical area (posterior limbic cortex) yielded no systematic acquisition-related unit response alterations.
\end{abstract}

There is great current interest in the electrophysiological correlates of learning (see reviews by John, 1967; Kandel \& Spencer, 1968; Thompson, Patterson, \& Teyler, 1972). A significant portion of this research has involved simultaneous recording of behavior and multiple-unit (e.g., Buchwald, Halas, \& Schramm, 1966; Cassady, Cole, Thompson, \& Weinberger ${ }^{1}$ ) or single-unit (e.g., Hirano, Best, \& Olds, 1970; Olds, Disterhoft, Segal, Kornblith, \& Hirsh, 1972; Segal \& Olds, 1972; Segal, Disterhoft, \& Olds, 1972; Woody, Vassilevsky, \& Engel, 1970) neuronal activity during conditioning of chronically implanted animals. In the present study, we have adopted an approach methodologically similar to that employed in these recent studies. Specifically, we have recorded multiple-unit activity from the cerebral cortex of the rabbit during signaled avoidance behavior. The rabbit was chosen as an experimental $\mathrm{S}$ because of behavioral data indicating the feasibility of obtaining reliable asymptotic conditioned response (CR) performance within a single conditioning session (Gabriel, 1968, 1970; Gabriel \& Vogt, 1972).

We would like to emphasize the necessity for caution in any attempt to demonstrate an electrophysiological representation of associative learning. Hall and Mark (1967) have shown that occurrence of behavioral

\footnotetext{
*Supported in part by Research Grant NS07661 from the National Institutes of Health (R.F.T.), Research Grant MH19314 from the National Institute of Mental Health (R.F.T.), Research Scientist Award MH06650 from the National Institute of Mental Health (R.F.T.), Postdoctoral Fellowship MH44158 from the National Institute of Mental Health (M.G.), and National Institute of Mental Health Training Grant MH11095 (W.W.). We wish to thank Shirley J. Adams and Fe E. Glanzman for technical assistance.

†Current address: Department of Psychology, Pomona College, Pomona, California 91711. Reprint requests should be sent to this address.
}

conditioning does not insure that correlated electrophysiological changes will necessarily reflect the associative processes underlying conditioning. Rather, many CNS response alterations seen during conditioning may be attributed to alterations in general arousal, fear, and other factors (see discussion by Thompson, Patterson, \& Teyler, 1972).

The strategy for dealing with this problem requires controls, such as unpairing or backward pairing of CS and UCS, for nonassociative effects (Rescorla, 1967). However, in view of the possible subtlety of processes underlying conditioning, the possible anatomical variation in the location of the neural elements involved in these processes as a function of constitutional factors and experience, and the difficulty in duplicating exactly electrode position from animal to animal, there are difficulties with these control procedures. For these reasons, it is desirable to control for nonassociative neural changes within the individual S. In the present experiments, we have approached the problem by adopting the criteria of acquired stimulus control. Specifically, this means that the measured response, whether behavioral or electrophysiological, must show: (a) a change over conditioning trials (acquisition), and (b) acquired stimulus specificity. The latter is evaluated by showing a decrease in responding due to alteration of the original CS. In other words, stimulus specificity denotes a significant stimulus generalization gradient with peak responsiveness at whichever stimulus is selected as the CS for conditioning. The two criteria of stimulus control are basically those stated by other authors (e.g., Ganz, 1968; Baron, 1965; Terrace, 1966; Jenkins \& Sainsbury, 1969).

We suggest that data demonstrating alterations in multiple-unit responses meeting these two criteria, when coupled with a behavioral demonstration of stimulus control, represent a viable approach to the analysis of associative events in the CNS. This approach becomes 
AN DN PN

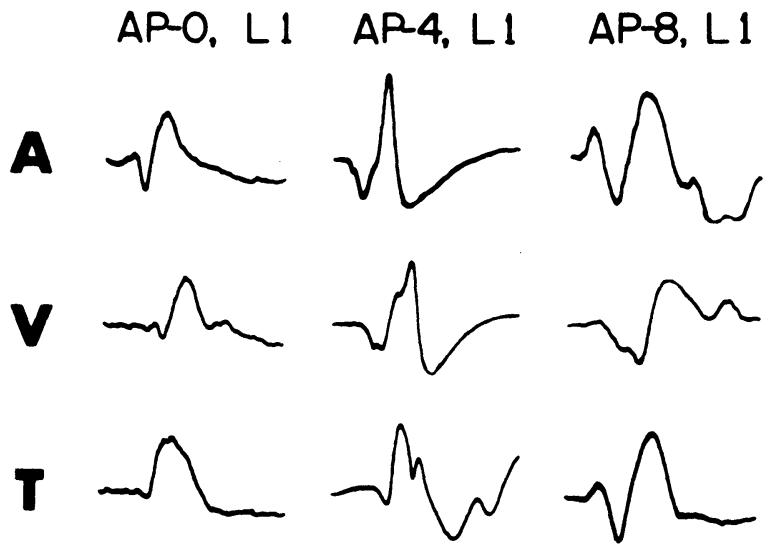

I $100 \mu v$.

Fig. 1. Representative gross evoked potentials recorded from the three nonspecific cortical areas (AN, DN, and PN) of the present study. The potentials were recorded from rabbits anesthetized with $\propto$-chloralose, using bipolar concentric (surface to depth) stainless steel electrodes. Upward deflection indicates a surface positive response polarity. The horizontal dimension of the figure represents the three nonspecific cortical areas and their respective stereotaxic surface coordinates $(\mathrm{AP}-\mathrm{O}=$ bregma $)$. The figure illustrates responsiveness of each area to three stimuli: (a) auditory click (A); (b) light flash (V); and (c) subcutaneous electric shock to the ipsilateral hindpaw (T).

useful if area-specific changes in neuronal activity can be shown during conditioning and, particularly so, if these changes can be shown to be stimulus specific in some areas and not in others. Recent work by Olds and his associates (above references) represents an example of this type of analysis.

With this approach in mind, we chose to focus initially upon an electrophysiological analysis of three "nonspecific" polysensory cortical areas in the rabbit. These areas were chosen because of studies indicating the involvement of nonspecific cortical "association areas" of the cat in the mediation of several phenomena of behavioral plasticity (Thompson \& Kramer, 1965; Thompson \& Shaw, 1965; Thompson \& Smith, 1967). The specific goal of the present experiment is to demonstrate acquisition of behavioral and cortical multiple-unit responses during avoidance conditioning. A second paper (Gabriel, Wheeler, \& Thompson ${ }^{2}$ ) will present data on the second criterion of stimulus control, that of acquired stimulus specificity.

\section{METHODS}

\section{Subjects and Apparatus}

Thirty-six naive male albino rabbits (Oryctolagus cuniculus), weighing from 3.5 to $4.0 \mathrm{lb}$, were obtained from a local supplier. All rabbits received avoidance conditioning in an automated Brogden-Culler rotating-wheel conditioning apparatus (Brogden \& Culler, 1936). The rotator was housed in an electrically

shielded chamber located in a room that was separate from but adjacent to the room containing the remaining apparatus. During conditioning, the rabbit was visible through one-way windows located in the shielded chamber and in the partition separating the rooms.

The conditioned stimulus (CS) was a $4,000-\mathrm{Hz}$ tone (10-msec rise time) produced by a sine-wave generator. The sine-wave generator output was fed through an attenuator, an electronic switch, and a power amplifier to a speaker located directly above the rotator. The intensity of the tone was set at $90 \mathrm{~dB}$ (re $.0002 \mathrm{dynes} / \mathrm{cm}^{2}$ ) for conditioning, an intensity that was $43 \mathrm{~dB}$ above the ambient noise level of the conditioning chamber. A shock unconditioned stimulus (UCS) was delivered through the grid floor of the rotator over a constant current $(2 \mathrm{~mA})$ circuit. The behavioral data were taken on a paper-tape event recorder.

Multiple-unit electrical activity of the cerebral cortex was recorded monopolarly from chronically implanted, large microelectrodes (15-25-micron tip diameter, 50-100 microns exposed, 700k-ohm impedence at $1 \mathrm{kHz}$ ) against an indifferent electrode placed over the frontal sinus. The recording electrodes were made from No. 3 stainless steel insect pins coated and baked with four coats of Epoxylite, and scraped at the tip. The multiple-unit activity was amplified by a two-stage circuit (Brakel, Babb, Mahnke, \& Verzeano, 1971). The first stage was a high-impedance input, low-impedance output system consisting of field-effect transistors soldered directly to an ITT Cannon strip connector. This assembly was embedded in Duratite 5-min epoxy at the rabbit's end of a shielded wire recording cable, which was connected to the field-effect transistor outputs. The cable was led through the axle of the rotator to battery-powered high-gain preamplifiers (second stage) located on a shelf within the conditioning chamber. During conditioning, the field-effect transistor input recording cable and the implanted electrodes were mated via another strip connector which had been affixed to the rabbit's skull in surgery. The two strip connectors were firmly locked together by a machine screw. This circuitry, the restricted range of movements allowed by the rotator, and an experimental design which made CS presentation contingent upon a lack of intertrial rotational responses, virtually eliminated movement artifact from the neural records (Gabriel $\left.{ }^{3}\right)$.

The outputs of the amplifiers were fed into the AM channels of a Crown-Vetter AM-FM tape recorder located in the adjacent room. Cortical multiple-unit activity was monitored continuously during the experiment, using a four-channel oscilloscope.

\section{Surgical Procedure}

The "nonspecific" cortical target areas of the present study are indicated in the left half of Fig. 6 . The three electrode targets lie $1 \mathrm{~mm}$ lateral to the midline, and are mutually separated by intervals of $4 \mathrm{~mm}$ in the anterior-posterior dimension. The areas are labeled $\mathrm{AN}, \mathrm{DN}$, and $\mathrm{PN}$ for anterior, dorsal, and posterior nonspecific cortex, respectively. AN corresponds to the area traditionally labeled motor-sensory cortex, whereas DN and PN fall within the bounds of rabbit limbic cortex (cf. Rose \& Woolsey, 1948). The designation of nonspecific is based upon the properties of evoked responses obtained from these areas in 25 rabbits anesthetized with $\propto$-chloralose. Each area manifested evoked responses to three stimuli: auditory click, light flash, and shock to the ipsilateral hindpaw. Representative gross evoked potentials to these stimuli are illustrated in Fig. 1 for each area in the chloralosed rabbit. The polysensory responsiveness, characteristics of waveform, waveform variability, and ordering of evoked response latencies to the different stimuli were similar to the same properties of association-evoked responses in the cat (Thompson, Johnson, \& Hoopes, 1963) and rat (Meikle, 1969).

Rabbits were acquired in groups of eight and were allowed acclimation to the vivarium conditions for at least $24 \mathrm{~h}$ prior to surgery. The surgery for a given group of rabbits was begun after a maximum period of 10 days following delivery of the rabbits. 

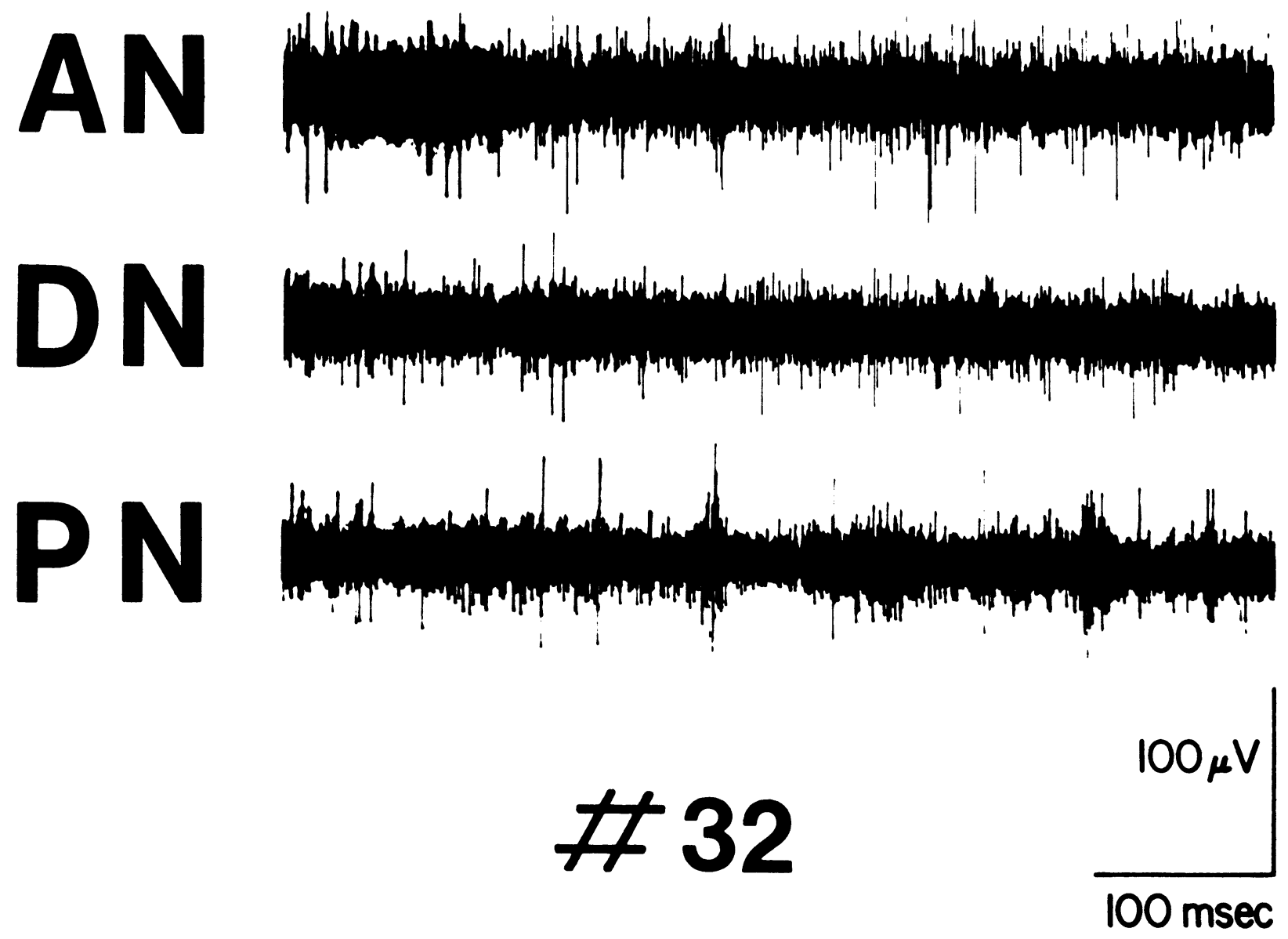

Fig. 2. Spontaneous multiple-unit activity recorded from the three nonspecific cortical areas (AN, DN, and PN) of Rabbit 32 immediately prior to the onset of acquisition trials.

A minimum of 7 days' recovery intervened between surgery and conditioning. The maximum recovery period was 12 days.

Each rabbit was anesthetized with $12.5 \mathrm{mg}$ of chlorpromazine followed by $25.0 \mathrm{mg}$ of sodium pentobarbital delivered through the marginal vein of the left pinna. The head was fixed into a rabbit head holder (Kopf Instruments Company), and the skull was exposed. Small holes were drilled through the skull over each cortical target area. Additionally, a hole was drilled over the frontal sinus, and the stainless steel screw reference electrode was threaded into place.

The electrodes were secured in position during implantation in the following manner. A 2-56 x 1/8 nylon pan-head screw, which had been drilled through its center using a .022-in.-diam bit, was threaded into each target hole through the skull. These nylon screws were advanced through the holes until their tips were flush with the undersurface of the skull. Once positioned, the screws were secured with dental acrylic. The electrodes were press fitted through the inner cylinders of these nylon screws and advanced through the dura matter into cortical tissue to a minimum depth of $1 \mathrm{~mm}$ below the surface. Fine-gauge stainless steel wires had been previously soldered to each electrode $7 \mathrm{~mm}$ above the recording tip, the remaining portion of the insect pin removed, and the solder joint coated with Insulex. Electrical activity was monitored continuously during advancement of the electrodes. Penetrations greater than $1 \mathrm{~mm}$ were made in some cases to obtain large-amplitude multiple-unit records. The wires from each electrode and from the frontal sinus reference were soldered to a 5-pin ITT Cannon strip connector, and the entire assembly was embedded in dental acrylic. The incision was closed around the assembly, and each rabbit was given a .5 -cc intramuscular injection of Bicillin (150,000 units).

Following the completion of behavioral testing, each rabbit was given an overdose of barbiturate, and small electrolytic lesions were placed at the tip of each electrode. The rabbit was perfused with saline followed by $10 \%$ Formalin, and then the brain was removed. Each brain was frozen and sectioned at 50 microns, and the sections containing electrode tracts were photographed while still wet (Fox \& Eichman, 1959). Subsequently, the sections were stained with cresyl-violet.

\section{Experimental Procedure and Design}

Each rabbit was given avoidance conditioning and overtraining with continuous recording of cortical multiple-unit activity. The onset of the CS preceded UCS onset by $5 \mathrm{sec}$, and both the CS and the UCS were response terminated. The maximum duration of the UCS was $1 \mathrm{sec}$. Response onset was defined as any rotation of at least $3 / 8 \mathrm{in}$. along the rotator's circumference. The intervals between cessation of a response and presentation of the CS (ITIs) were $10,15,20$, or $25 \mathrm{sec}$. These ITIs occurred in a randomized sequence throughout the experiment. A response in the presence of the CS (avoidance CR) terminated that stimulus and prevented UCS occurrence. Responses during the ITI reset the ITI. Conditioning was carried out to a criterion of 10 consecutive avoidance CRs. After attaining criterion, each $\mathrm{S}$ received 100 additional trials of conditioning (overtraining). During overtraining, the intensity of the CS varied in a restricted random sequence so that each of five CS intensities (80.85, 90, 


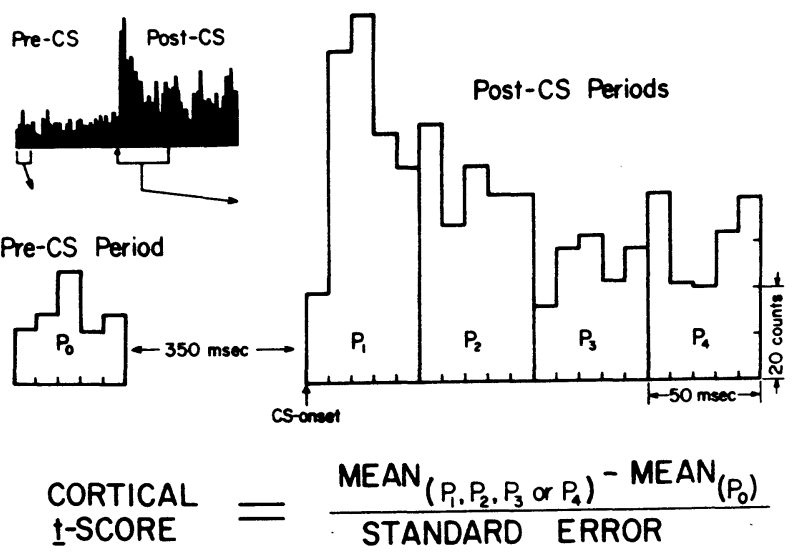

Fig. 3. Illustration of the computation of cortical $t$ scores. The small histogram, pictured in the upper left of the figure, illustrates unit counts from DN cumulated in successive 10 -msec bins during 20 postacquisition trials of a representative rabbit. The brackets underneath this histogram indicate the pre- and post-CS onset data segments used in the computation of cortical $t$ scores. The large histograms below and to the right are enlargements of these pre- and post-CS onset data segments, respectively. The upward-pointing arrows indicate CS onset in both the small and large plots. The post-CS activity was divided into four consecutive 50 -msec periods $\left(P_{1}, P_{2}, P_{3}\right.$, and $\left.P_{4}\right)$, beginning at $C S$ onset. The mean number of counts in each bin was computed for each post-CS-onset period. Likewise, the mean and standard deviation of the five bins within the pre-CS period $\left(\mathbf{P}_{0}\right)$ was computed. The standard deviation of $\mathbf{P}_{0}$ was taken as the best estimate of the variation in ongoing baseline activity and the standard error of the mean difference computed, using the denominator of the Student $t$ computational formula. Four separate cortical $t$ scores were obtained for each histogram in acquisition and overtraining, using the formula at the bottom of the figure.

95 , and $100 \mathrm{~dB}$ ) occurred four times in each 20-trial block. This was done to control for potential perceptual intensity differences during a subsequent test for stimulus generalization to CS frequency (Gabriel, Wheeler, \& Thompson ${ }^{2}$ ). All conditioning and overtraining was completed in a single experimental session.

\section{Data Analysis}

The behavioral CRs of the 36 rabbits were analyzed using standard techniques. Ten of the rabbits showing large-amplitude multiple-unit records ( 50 microV peak-to-peak minimum) in all three cortical areas were selected for electrophysiological analysis. Figure 2 shows spontaneous multiple-unit activity from one of these Ss. Further selection within this subset was based upon the histological verification of the presence of electrode tips within cortical gray matter (see histology section of Results).

Tapes of the multiple-unit records were played through high-pass filters $(500-10,000 \mathrm{~Hz})$ and through notch filters. The notch filters were set to the CS frequency $(4 \mathrm{kHz})$ to eliminate possible volume conducted cochlear microphonic produced by the CS. The notch filters were used for the records of all three cortical areas and were found to be completely effective in eliminating CS-frequency components from the neural records.

Output of the notch filters was fed into a monitoring oscilloscope and into Schmidt triggers. The latter were adjusted independently for each cortical area to yield 200-300 output pulses/sec during the non-CS periods. With this procedure, all trigger settings fell within a range of +10 to +15 microV above zero. The trigger settings were well above the electronic noise levels for all records. The records and corresponding Schmidt trigger pulses were monitored continuously during playback. Output from the Schmidt triggers was fed into a Digital Equipment PDP-12 computer, which counted the number of Schmidt trigger pulses and stored the counts into consecutive 10 -msec bins preceding and following CS onset. The counting was performed simultaneously for all electrodes. Time histograms were compiled by cumulating counts over corresponding bins of consecutive trials (CS presentations). For acquisition, the number of trials in each histogram represented $1 / 10$ of the total number of precriterial trials for a given $S$. Five histograms, each composed of 20 trials, were compiled using the 100 postcriterial overtraining trials. A preliminary analysis of the behavioral results showed that the shortest latency of behavioral response was greater than $.5 \mathrm{sec}$. Therefore, the period of electrophysiological analysis was restricted to the initial $200 \mathrm{msec}$ following CS onset to avoid movement artifact.

Numerical scores which reflected the magnitude of post-CS onset multiple-unit responses were computed for each histogram of conditioning and overtraining. The computation of these scores (cortical t scores) is illustrated in Fig. 3 for an overtraining histogram obtained from DN. [The response seen in this histogram was not present before training (see Results).] The mean number of counts in five consecutive 10 -msec pre-CS bins $\left(P_{0}\right)$ was subtracted from the means of four sets of five consecutive 10 -msec bins just following CS onset $\left(P_{1}, P_{2}, P_{3}\right.$, and $\left.P_{4}\right)$. Each of the four mean differences was divided by the standard error of the mean difference. The standard error was computed using the standard deviation of the pre-CS bins $\left(\mathrm{P}_{0}\right)$ as the best estimate of ongoing variation in spontaneous baseline activity. Thus, four cortical $t$ scores, representing multiple-unit activity in four consecutive $50-\mathrm{msec}$ periods following CS onset, were obtained for each histogram. Each score may be referred to the $t$ distribution (two-tailed, $d f=8$ ). Cortical $t$ scores equal to or greater than 2.306 represent statistically reliable evoked responses $(p<.05)$. This normalization procedure tends to control for possible changes in multiple-unit activity as a function of ongoing behavioral reactivity (see Imig \& Weinberger, 1970).4

\section{RESULTS}

\section{Behavioral CRs, All Ss}

The percentage of behavioral CRs in each fifth of conditioning and in each of five 20-trial blocks of

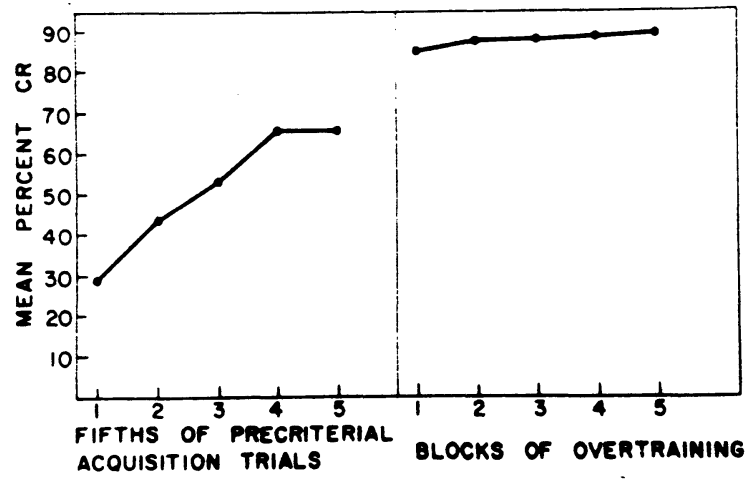

Fig. 4. Mean percent of conditioned avoidances (CR) as a function of fifths of precriterial acquisition trials and 20-trial blocks of postcriterial overtraining for 36 rabbits. Acquisition and overtraining trials are discontinuously plotted since the data from the 10-trial criterial run are not included. 


\section{AN}

DN

PN

Fig. 5. Photographs of representative cresyl-violet-stained coronal sections through $\mathrm{AN}, \mathrm{DN}$, and $\mathrm{PN}$, respectively. An arrow indicates the position of an electrode tip within each section.
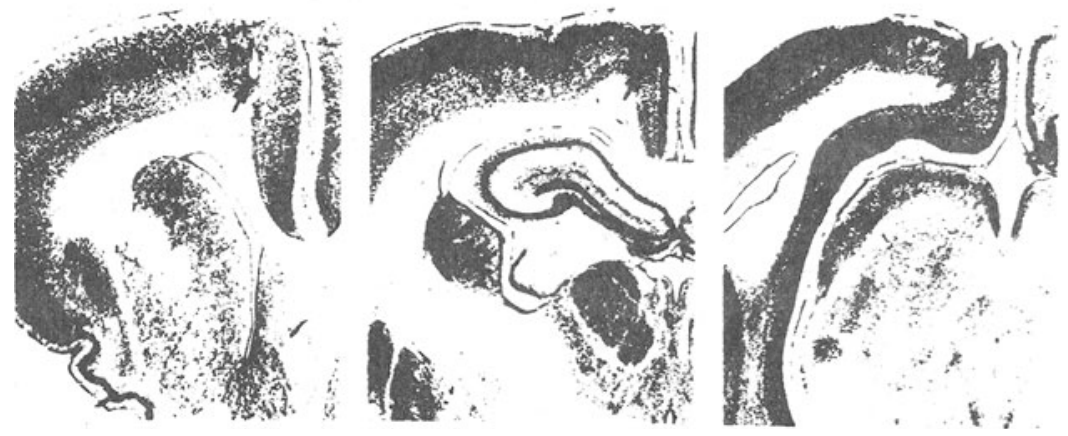

overtraining is shown in Fig. 4 for all 36 rabbits. The mean number of trials prior to criterion was 52.75. A repeated measures analysis of variance yielded a significant effect of fifths $(F=21.10, d f=4 / 140$, $\mathrm{p}<.001)$ of conditioning. No significant effect of overtraining blocks was obtained. These results corroborated those obtained from previous studies (Gabriel, 1968, 1972), in which similar behavioral procedures were used, indicating (a) single-session acquisition of the avoidance $\mathrm{CR}$, and (b) asymptotic performance of the CR during 100 postcriterial overtraining trials.

\section{Histology, Selected Ss}

Electrophysiological data from the 10 selected rabbits are reported only for those electrode placements where the tips were shown to lie in Layers II, III, IV, or V of the cortical gray matter. Figure 5 shows a coronal section through each target area (AN, DN, and PN), illustrating the tip positions of representative, histologically acceptable, electrodes (cresyl-violet stain). This further selection, based on histological data, resulted in the following electrode Ns for each cortical area: $\mathrm{N}(\mathrm{AN})=9, \mathrm{~N}(\mathrm{DN})=10, \mathrm{~N}(\mathrm{PN})=8$. All of the 10 rabbits had at least two electrodes meeting the histological criterion. The right half of Fig. 6 shows a scatter diagram of the electrode positions. Those electrodes within $\mathrm{AN}$ are confined to the area traditionally labeled motor-sensory cortex, whereas those within DN and PN fall inside the bounds of rabbit limbic cortex (cf. Rose \& Woolsey, 1948). Additionally, all of the electrode placements fell within the confines of those areas shown to be "nonspecific" in anesthetized preparations (see Methods).

\section{Behavioral and Cortical Responses, Selected Ss}

In order to maximize the probability of detecting any reliable changes in cortical activity from the 10 selected rabbits during the acquisition phase of conditioning, statistical comparisons were made between the data obtained from the first and second halves of precriterial trials. Accordingly, a repeated measures analysis of variance was performed on halves of acquisition (first half vs second half) for behavioral CRs, using the data of

\section{ELECTRODE TARGETS}

A

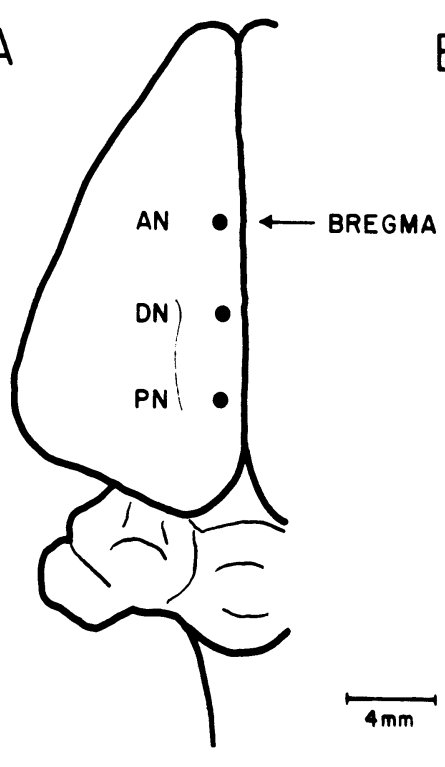

ELECTRODE PLACEMENTS
Fig. 6. Location of cortical target areas (A) and the surface topography of electrode placements (B). The left portion of this figure (A) shows the location of the three nonspecific cortical target areas within a diagram of the left cerebral hemisphere of the rabbit. The target areas are labeled AN, DN, and PN for anterior, dorsal, and posterior nonspecific cortex, respectively. The right portion shows a scatter diagram of the actual electrode positions for the Ss used in the electrophysiological analysis. The criteria for selection of $\mathrm{Ss}$ and electrodes are reported in the main text. 


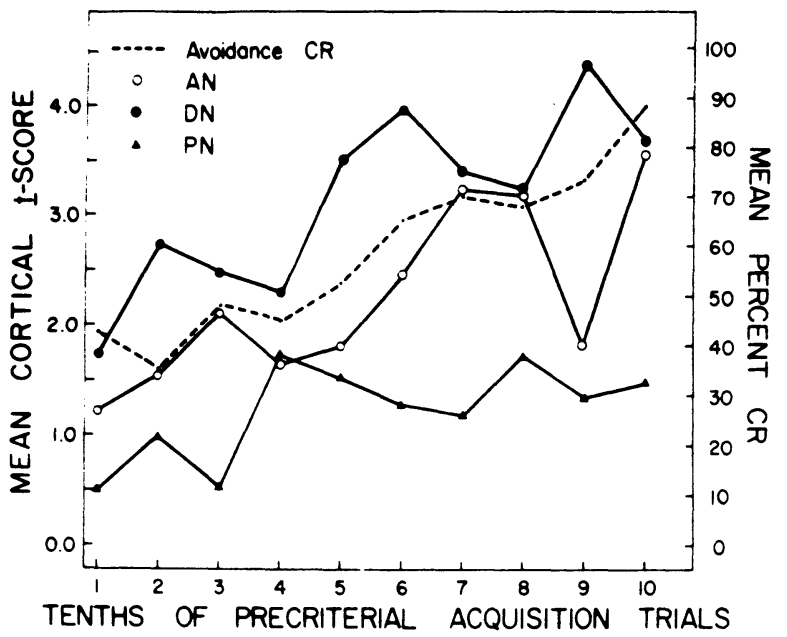

Fig. 7. Mean percent conditioned avoidances (CR, dashed line) and mean cortical $t$ scores obtained from the first 50 -msec period following CS onset for AN (circles), DN (filled circles), and PN (triangles) as a function of 10ths of precriterial acquisition trials for the subset of 10 rabbits. Each mean score for $A N$, DN, and PN represents an $N$ of 9,10 , or 8 individual scores, respectively.

the selected subset of $10 \mathrm{Ss}$. Behavioral CRs showed a significant increase over halves $(F=18.99, \mathrm{df}=1 / 9$, $\mathrm{p}<.001)$. An identical analysis of variance was applied to the cortical $t$ scores of each of the four post-CS-onset periods for each of the electrode target areas (i.e., a separate two-level analysis was done for each period). The cortical t scores showed a significant increase over halves for $\mathrm{AN}(\mathrm{F}=11.06, \mathrm{df}=1 / 8, \mathrm{p}<.025)$ and for $\mathrm{DN}(\mathrm{F}=7.91, \mathrm{df}=1 / 9, \mathrm{p}<.025)$ only for the initial 50 -msec period following CS onset. None of the remaining $50-\mathrm{msec}$ periods or the remaining area (PN) showed a significant effect of conditioning.

The acquisition data for the first $50-\mathrm{msec}$ analysis period are plotted in Fig. 7, showing mean cortical $t$ scores from AN, DN, and PN as a function of 10ths of precriterial acquisition trials. This figure illustrates the significant increase in responsiveness over trials seen in $\mathrm{AN}$ and DN, contrasted to an absence of significant change in PN. The behavioral acquisition function (CRs) for the subset of $10 \mathrm{Ss}$ is also plotted in this figure.

Figure 8 illustrates the acquisition effect in terms of the "raw" multiple-unit records from Rabbit 34. Individual traces from every eighth precriterial acquisition trial are shown from AN, DN, and PN. The behavioral criterion of 10 consecutive avoidances was reached at Trial 77. This figure shows the clear development of a CS-evoked multiple-unit response in $\mathrm{AN}$ and DN. This response was not present in the initial phases of CS-UCS pairing. Figure 9 shows four traces from the three areas of the same animal representing unit responses during Trials $1,26,51$, and 76 of overtraining. The conditioned multiple-unit responses seen in AN and DN were consistently present during overtraining.
The records from AN and DN showed the effects of conditioning in every rabbit subjected to an electrophysiological analysis. There was little or no indication of unit responsiveness during the initial trials of conditioning. However, as CS-UCS pairing progressed, clear CS-evoked multiple-unit responses developed in AN and DN. The raw records indicated that the onset latency of the acquired CS-evoked responses seen in AN and DN ranged from 15 to $25 \mathrm{msec}$.

Figure 10 demonstrates the same effect using histograms obtained from Rabbit 32 . Each histogram is composed of 80 sequential $10-\mathrm{msec}$ bins (40 pre-CS, 40 post-CS onset) in order to clearly illustrate both typical variation in pre-CS spontaneous activity and the pattern of the post-CS-onset response. The first 20 bins following the CS-onset indicator in each histogram represent the $200-\mathrm{msec}$ period for which four cortical $t$ scores were computed. The first two histograms from each area represent the first and second half of acquisition, respectively. Each of these histograms contains the total counts per bin cumulated over sequential CS presentations (criterion reached at Trial 30). The histograms from both AN and DN show an increased response to the CS during the second half of acquisition. PN also shows some indication of a response; however, it does not appear to increase relative to the first half. The next five histograms from each area are composed of activity cumulated from the five 20 -sequential-trial blocks of overtraining. These overtraining histograms show a clear post-CS-onset multiple-unit response in AN and DN. Some indication of responsiveness is also seen in PN during overtraining of this rabbit.

In order to assess possible change in the evoked neuronal activity during overtraining, a repeated measures analysis of variance was computed for the cortical $t$ scores obtained from the five 20-trial overtraining histograms. Also, given the relatively large number of conditioning trials of overtraining, the data provided an opportunity for analysis of the post-CS-onset neuronal response pattern. Thus, blocks of overtraining (five levels) and $50-\mathrm{msec}$ post-CS-onset periods (four levels) were orthogonal factors of the present analysis. Two cortical areas (AN and DN) yielded main effects for the periods factor. None of the remaining main effects or interaction effects were significant, and no significant effects were obtained from PN. The absence of a significant effect of blocks of overtraining in any cortical area suggested no systematic change in the magnitudes of cortical $t$ scores as a function of stage of overtraining.

The significant effect of post-CS-onset periods is illustrated in Table 1, which shows the overall overtraining mean cortical $t$ scores for each period in each cortical area, along with the corresponding $F$ ratios and probability levels for the periods factor. In order to determine the nature of the periods effect shown in AN and DN, two-tailed t tests carried out at the .05 level of 


\section{\# 34}

TRIAL

AN

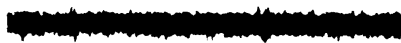

$\Delta$
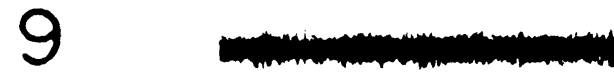

$\Delta$

17

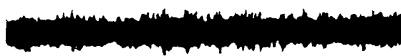

$\Delta$

25

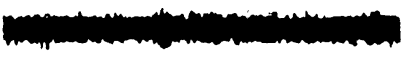

$\Delta$

33

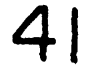

49

57

65

73
ACQUISITION
PN

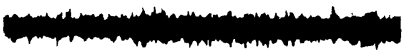

$\Delta$

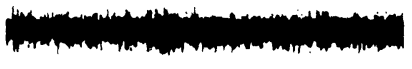

$\Delta$

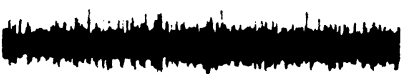

$\Delta$

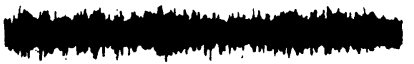

$\Delta$

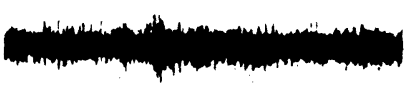

$\Delta$

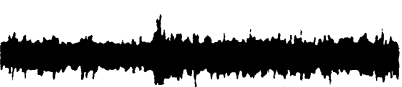

$\Delta$
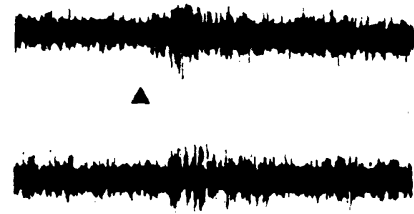

$\Delta$

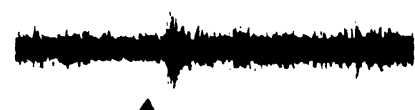

$\Delta$

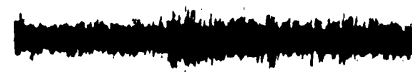

$\Delta$

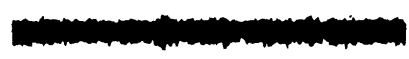

$\Delta$
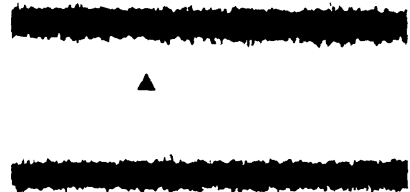

$\Delta$

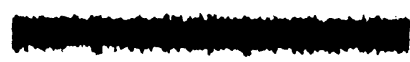

$\Delta$

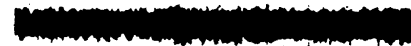

$\Delta$

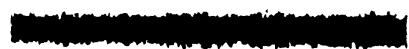

$\Delta$

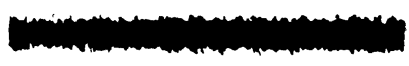

$\Delta$
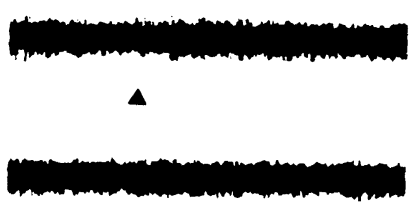

$\Delta$

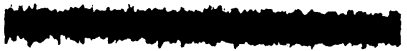

$\Delta$

\section{$\Delta=$ CS-onset}

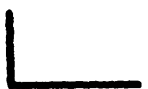

Fig. 8. Raw multiple-unit records from AN, DN, and PN of Rabbit 34, representing every eighth precriterial acquisition trial (criterion reached at Trial 77). Each trace is $300 \mathrm{msec}$ long and includes $100 \mathrm{msec}$ of pre-CS-onset activity. Triangles indicate CS onset. The horizontal and vertical calibration lines represent $100 \mathrm{msec}$ and 100 microV, respectively. 


\section{\# 34 OVERTRAINING}

\section{TRIAL}

AN

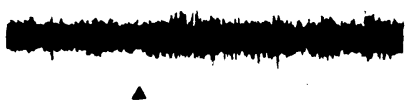

26

51

76
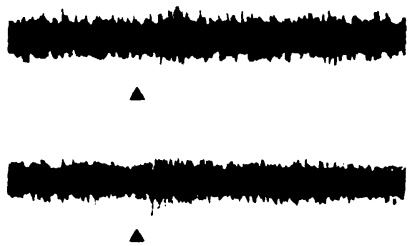

\section{DN}

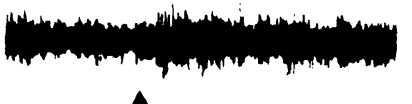

$\Delta$

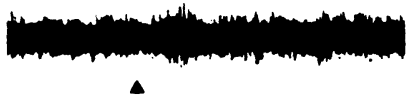

$\Delta$

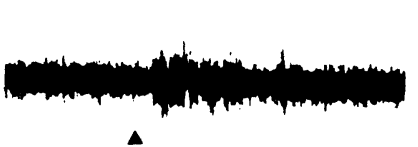

$\Delta$

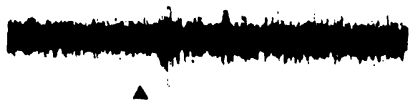

$\Delta$

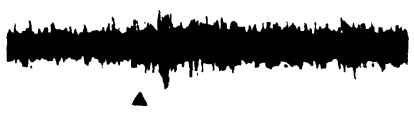

PN
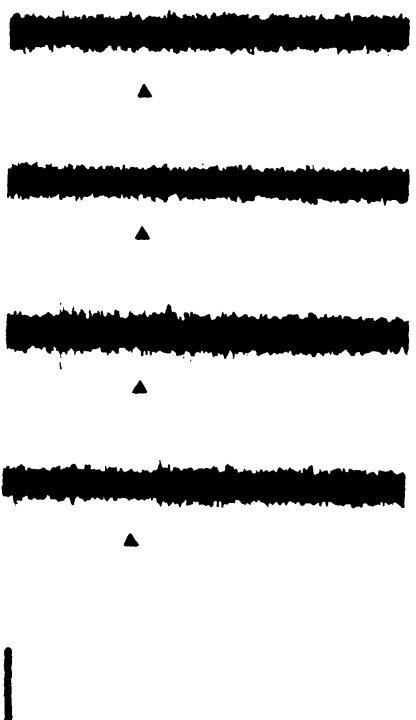

\section{$\Delta=$ CS-onset}

Fig. 9. Raw multiple-unit records from AN, DN, and PN of Rabbit 34, representing Trials 1, 26, 51 , and 76 of overtraining. Each trace is $300 \mathrm{msec}$ long and includes $100 \mathrm{msec}$ of pre-CS-onset activity. Triangles indicate CS onset. The horizontal and vertical calibration lines represent $100 \mathrm{msec}$ and 100 microV, respectively.

significance were applied to the means of each period subsequent to the $F$ tests. The results of these subsequent tests showed a significantly greater cortical $t$ score for Period 1 within AN compared to the statistically homogeneous means of the remaining periods of this cortical area. There were no significant differences between the period means within DN. These results suggested that CS-evoked cortical activity recorded from $\mathrm{DN}$ during asymptotic $\mathrm{CR}$ performance was sustained throughout the total 200-msec period of analysis, whereas evoked activity within $\mathrm{AN}$ was greater in the initial $50-\mathrm{msec}$ period compared to the remaining three periods. In addition, it should be mentioned that a tendency for maintenance over the four 50-msec periods of the evoked activity within AN was suggested by the relatively large mean cortical $t$ scores for Periods 2,3 , and 4 , each exceeding 2.306. Thus, the two nonspecific cortical areas showing significant increases in CS-evoked activity during avoidance acquisition also appeared to produce sustained responding to the CS during overtraining. These effects stand in contrast to the lack of statistical evidence for acquisition-related increase in responsiveness in $\mathrm{PN}$, although significant mean cortical $\mathrm{t}$ scores for Periods 1 and 2 were observed when the data of 100 postcriterial overtraining trials were combined for all Ss.

\section{DISCUSSION}

The present study demonstrated the simultaneous acquisition of behavioral and cortical multiple-unit responses during single-session avoidance conditioning. The behavioral results showed a significant acquisition effect. Cortical $t$ scores (indices of the magnitude of CS-evoked multiple-unit responses) from two nonspecific cortical areas (AN and DN) showed a significant increase in magnitude over conditioning trials paralleling the behavioral acquisition function. No increase was observed for the cortical $t$ scores of a third nonspecific area (PN) during the acquisition phase of conditioning. Thus, the behavioral and cortical data of $\mathrm{AN}$ and DN satisfied the initial criterion of acquired stimulus control (e.g., acquisition).

The acquisition-related increase in magnitude of multiple-unit responses shown for $\mathrm{AN}$ and DN was restricted to the initial $50 \mathrm{msec}$ of neural activity following CS onset. The onset latency of these responses ranged from 15 to $25 \mathrm{msec}$. These findings, therefore, 
\#32

\section{Acquisition}

\section{AN}

DN

PN

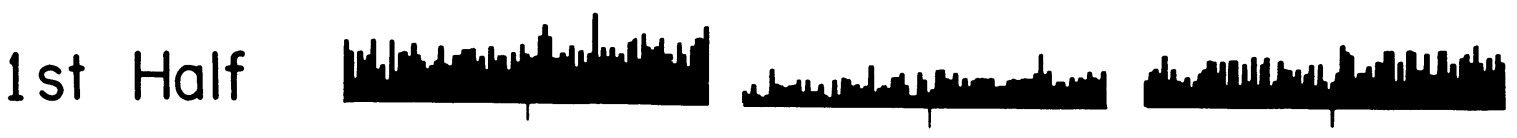

2nd Half

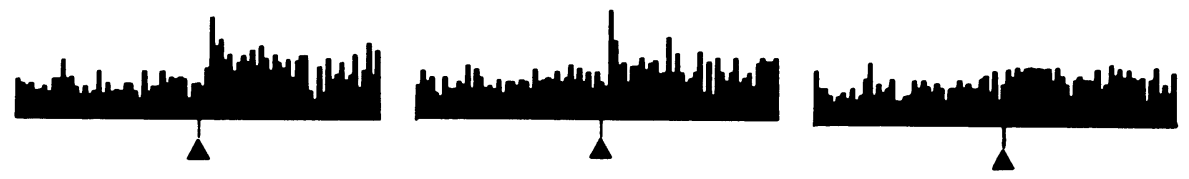

\section{Overtraining}

Block 1

Block 2

Block 3

\section{Block 4}

Block 5
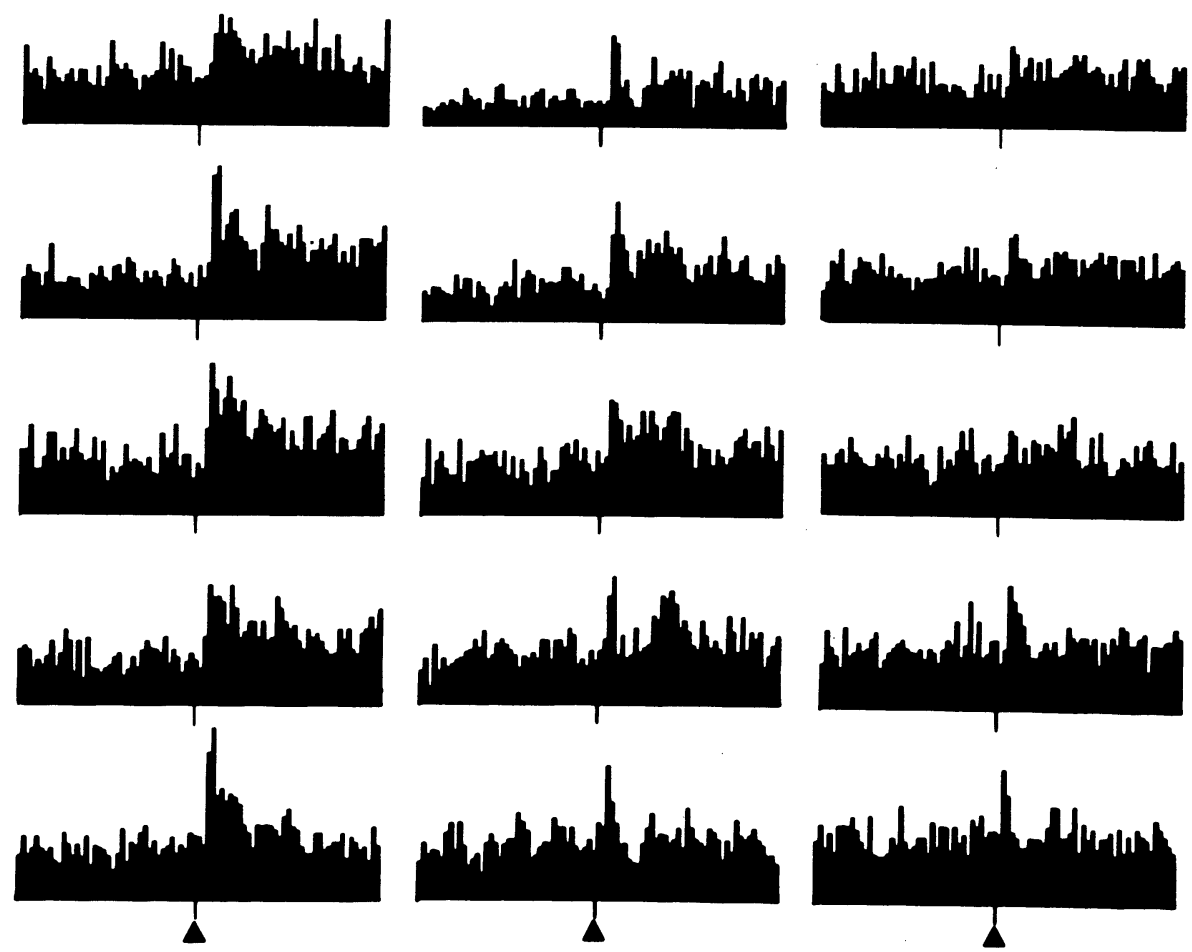

$$
\Delta=\text { CS-onset }
$$

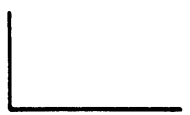

Fig. 10. Histograms from AN, DN, and PN of Rabbit 32 obtained during halves of acquisition and the five blocks of overtraining. Each histogram is composed of pre- and post-CS onset activity collected into 80 sequential 10 -msec bins. Each acquisition histogram represents cumulated counts from 15 individual trials (criterion reached at Trial 30 for Rabbit 32). Each overtraining histogram contains 20 cumulated sequential overtraining trials. The triangles indicate CS onset. The horizontal and vertical calibration lines represent $400 \mathrm{msec}$ and 50 counts, respectively. 
Table 1

Mean Cortical t Score for Consecutive 50-msec Post-CS Onset Periods

\begin{tabular}{|c|c|c|c|c|c|}
\hline \multirow[b]{2}{*}{ Area } & \multicolumn{4}{|c|}{ Periods } & \multirow[b]{2}{*}{ F Ratios } \\
\hline & 1 & 2 & 3 & 4 & \\
\hline AN & 7.57 & 4.91 & 4.36 & 3.62 & $\begin{array}{l}F=28.36 \\
d f=3 / 24 \\
p>.001\end{array}$ \\
\hline $\mathrm{DN}$ & 6.49 & 5.64 & 4.85 & 4.14 & $\begin{array}{l}F=3.82 \\
d f=3 / 27 \\
p>.025\end{array}$ \\
\hline PN & 3.14 & 2.76 & 2.24 & 2.15 & $\begin{array}{l}F=2.36 \\
d f=3 / 21 \\
p \cong 10\end{array}$ \\
\hline
\end{tabular}

agree with the data of Olds and his associates (Olds et al, 1972), showing short-latency changes in CS-correlated single-unit activity (less than $20 \mathrm{msec}$ in the rat) during conditioning. The absence of a simultaneous, significant alteration in the cortical $t$ scores from PN suggested anatomical differentiation of the cortical conditioning effect, a finding also in agreement with the work of Olds et al (1972).

The rabbits of the present study showed rapid acquisition of the avoidance $\mathrm{CR}$ and maintained avoidance behavior throughout the 100-trial overtraining period. The cortical responses of $\mathrm{AN}$ and DN also showed maintenance throughout the overtraining period. In addition, PN showed indication of responsiveness in overtraining despite absence of a precriterial acquisition effect. The cortical $t$ scores of the first and second post-CS-onset periods exceeded the critical level when the total 100 overtraining trials were combined. This finding may reflect a genuine acquisition-related change in PN responsiveness; however, the lack of a significant blocks effect for PN during overtraining mitigates this possibility. Alternatively, PN may show small multiple-unit responses throughout training which are not acquisition related and are not statistically detectable during acquisition due to the small number of individual trials combined in each histogram. Visual examination of Fig. 10 suggests that this may be the case.

A difference between the response pattern of AN vs DN was suggested from the analysis of the post-CS-onset periods of the overtraining histograms. The magnitude of the mean cortical $t$ score obtained from $\mathrm{AN}$ was greater in the initial 50-msec period, compared to the remaining three periods, although all periods showed significant scores in AN. In contrast, DN showed no reliable difference in the mean magnitudes of the cortical $t$ scores for the different periods, with all four periods showing highly reliable responses. These results suggested that the evoked multiple-unit response of DN is sustained over the full $200-\mathrm{msec}$ analysis interval. On the other hand, the response in AN was phasic, declining in magnitude for the later $50-\mathrm{msec}$ periods, compared to the initial one.
Data from the same and additional Ss will be presented in a subsequent paper (Gabriel, Wheeler, \& Thompson ${ }^{2}$ ) which satisfy the second criterion of acquired stimulus control, that of acquired stimulus specificity. Briefly, the rabbits were tested for stimulus generalization to tone frequency in an extinction paradigm on the test day following conditioning. A behavioral stimulus generalization gradient with peak responsiveness to the $4-\mathrm{kHz} \mathrm{CS}$ was shown. Likewise, the magnitude of cortical t scores from DN was greater to the $4-\mathrm{kHz}$ tone used as the $\mathrm{CS}$ than to generalization tones. The records from $\mathrm{AN}$ and $\mathrm{DN}$ did not show gradients, suggesting that not all cortical areas show these effects. Additional rabbits trained with other CSs in an identical paradigm also showed peak neural responsiveness in DN to the CS during generalization testing, indicating that these effects are training specific.

In conclusion, it is possible, on the basis of the present acquisition and overtraining results, to assume partial fulfillment of the criteria of associative learning which we have adopted, namely those of acquired stimulus control. Specifically, the initial (acquisition) criterion was satisfied in terms of behavioral and cortical measures. In a subsequent paper, the acquired stimulus specificity of the behavioral and multiple-unit responses showing acquisition will be examined in greater detail (Gabriel, Wheeler, \& Thompson ${ }^{2}$ ).

\section{REFERENCES}

Baron, M. R. The stimulus, stimulus control and stimulus generalization. In D. I. Mostovsky (Ed.), Stimulus generalization. Stanford, Calif: Stanford University Press, 1965.

Brakel, S., Babb, T., Mahnke, J., \& Verzeano, M. A compact amplifier for extra cellular recording. Physiology \& Behavior, 1971, 6, 731-733.

Brogden, W. J., \& Culler, E. A. Device for motor conditioning of small animals. Science, 1936, 83, 269.

Buchwald, J. S., Halas, E. S., \& Schramm, S. Changes in cortical and subcortical unit activity during behavioral conditioning. Physiology \& Behavior, 1966, 1, 11-22.

Fox, C. A., \& Eichman, J. A rapid method for locating intracerebral electrode tracks. Stain Technology, 1959, 34, 39-42.

Gabriel, M. Effects of intersession delay and training level in avoidance extinction and inter-trial behavior. Journal of Comparative \& Physiological Psychology, 1968, 66, 412-416.

Gabriel, M. Intersession exposure to conditioning apparatus, avoidance extinction and intertrial behavior. Journal of Comparative \& Physiological Psychology, 1970, 72, 244-249.

Gabriel M., \& Vogt, J. Incubation of avoidance CRs in the rabbit produced by increase over time in stimulus generalization to apparatus. Behavioial Biology, 1972, 7, 113-126.

Ganz, L. An analysis of generalization behavior in the stimulus deprived organism. In G. Newton and S. Levine (Eds.), Early experience and behavior. Springfield, Ill: Thomas, 1968.

Hall, R. D., \& Mark, R. G. Fear and modification of acoustically evoked potentials during conditioning. Journal of Neurophysiology, 1967, 30, 893-910. 
Hirano, T., Best, P., \& Olds, J. Units during habituation, discrimination learning and extinction. Electroencephalography \& Clinical Neurophysiology, 1970, $28,127-135$.

Imig, T. J., \& Weinberger, N. M. Auditory system multi-unit activity and behavior in the rat. Psychonomic Science, 1970, $18,164-165$.

Jenkins, H. M., \& Sainsbury, R. S. The development of stimulus control through differential reinforcement. In N. J. McIntosh and W. K. Honig (Eds.), Fundamental issues in associative learning. Halifax: Dalhousie University Press, 1969.

John, E. R. Mechanisms of memory. New York: A cademic Press, 1967.

Kandel, E. R., \& Spencer, W. A. Cellular neurophysiological approaches in the study of learning. Physiology \& Behavior, $1968,48,65-134$.

Meikle, M. B. Response properties of single neurons in a nonspecific polysensory area of the cerebral cortex of the rat. Unpublished doctoral dissertation. University of Oregon Medical School, 1969.

Olds, J., Disterhoft, J., Segal, M., Kornblith, D. L., \& Hirst, R. Learning centers of rat brain mapped by measuring latencies of conditioned unit responses. Journal of Neurophysiology, 1972, 35, 202-219.

Rescorla, R. A. Pavlovian conditioning and its proper control procedures. Psychological Review, 1967, 74, 71-80.

Rose, J. E., \& Woolsey, C. N. Structure and relations of limbic cortex and anterior thalamic nuclei in rabbit and cat. Journal of Comparative Neurology, 1948, 89, 279-348.

Segal, M., Disterhoft, J., \& Olds, J. Hippocampal unit activity during classical aversive and appetitive conditioning. Science, $1972,175,792-794$

Segal, M., \& Olds, J. Behavior of units in hippocampal circuit of rat during learning. Journal of Neurophysiology, 1972, 35, 680-690.

Terrace, H. S. Stimulus control. In W. K. Honig (Ed.), Operant behavior: Areas of research and application. New. York: Appleton-Century-Crofts, 1966

Thompson, R. F., Johnson, R. H., \& Hoopes, J. J. Organization of auditory, somatic sensory, and visual projection to association fields of cerebral cortex in the cat. Journal of Neurophysiolcgy, 1963, 26, 343-364.

Thompson, R. F., \& Kramer, R. F. Role of association cortex in sensory preconditioning. Journal of Comparative \& Physiological Psychology, 1965, 60, 186-191.

Thompson, R. F., Patterson, M. M., \& Teyler, T. J. The neurophysiology of learning. Annual Review of Psychology, 1972, 23, 73-104.

Thompson, R. F., \& Shaw, J. A. Behavioral correlates of evoked activity recorded from association areas of the cerebral cortex. Journal of Comparative \& Physiological Psychology, 1965, 60, 329-339.

Thompson, R. F., \& Smith, H. E. Effects of association area lesions on auditory frequency discrimination in cat. Psychonomic Science, 1967, 8, 123-124.

Woody, C. D., Vassilevsky, N. N., \& Engel, J., Jr. Conditioned eye blink: Unit activity at coronal precruciate cortex of the cat. Journal of Neurophysiology, 1970, 33, 851-864.

\section{NOTES}

1. Cassady, J. M., Cole, M., Thompson, R. F., \& Weinberger, N. M. Neural correlates of asymptotic avoidance and classical conditioned leg flexion. In preparation.

2. Gabriel, M., Wheeler, W., and Thompson, R. F. Multiple unit activity of the rabbit cerebral cortex during stimulus generalization of avoidance behavior. In preparation.

3. Gabriel, M. A system for multiple unit recording during avoidance behavior of the rabbit. In preparation.

4. Analogous methods of controlling for variation in background multiple-unit responsiveness were first developed in our laboratories more or less independently by $\mathbf{M}$. Cassady, working on a joint project with M. Cole, N. M. Weinberger, and R. F. Thompson (see Cassady et $\mathrm{al}^{\mathbf{1}}$ ). These procedures are basically the same as those developed, independently, by J. Olds and his associates (see Olds et al, 1972).

(Accepted for publication January 29, 1973.) 